Recepción: 19 / 11 / 2018

Aceptación: 28 / 12 / 2018

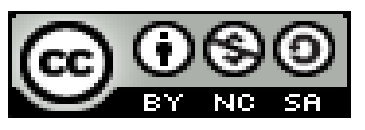

Ciencias Económicas

Publicación: 20 / 02 / 2019

Artículo de Investigación

\title{
Estrategias de competitividad para el fortalecimiento de las microempresas del cantón Jipijapa
}

\section{Competitiveness strategies for the strengthening of micro enterprises in the Jipijapa canton}

\section{Estratégias de competitividade para o fortalecimento de microempresas no cantão de Jipijapa}

\author{
Laura P. Muñiz-Jaime ${ }^{\mathrm{I}}$ \\ laura.muñiz@unesum.edu.ec \\ José A. Franco-Yoza II \\ jose.franco@unesum.edu.ec \\ Zoila M. Delgado-Gutierrez ${ }^{\text {III }}$ \\ zoila.delgado@unesum.edu.ec \\ Adela del Jesús Lucio-Pillasagua IV \\ adela.lucio@unesum.edu.ec \\ Carlos R. Tumbaco-Tumbaco V \\ carlosrenant@hotmail.com
}

\section{Correspondencia: laura.muñiz@unesum.edu.ec}

I. Magister en Contabilidad y Auditoría; Economista; Docente de la Universidad Estatal del Sur de Manabi, Jipijapa, Ecuador.

II. Magister en Contabilidad y Auditoría; Economista; Docente de la Universidad Estatal del Sur de Manabi, Jipijapa, Ecuador.

III. Magister en Contabilidad y Auditoria; Economista; Docente de la Universidad Estatal del Sur de Manabi; Jipijapa, Ecuador.

IV. Magister en Gerencia Educativa; Contador Publico; Ingeniera en Auditoria; Docente de la Universidad Estatal del Sur de Manabi; Jipijapa, Ecuador.

v. Economista de la Universidad Estatal del Sur de Manabi; Jipijapa, Ecuador. 
Estrategias de competitividad para el fortalecimiento de las microempresas del cantón Jipijapa

\title{
Resumen
}

En la presente investigación se analizaron las estrategias de competitividad y como su aplicación fortalece a las microempresas del cantón Jipijapa, esto debido al problema principal ¿Cómo las estrategias de competitividad aportan al fortalecimiento de las microempresas del cantón Jipijapa?, teniendo como objetivo general dicho estudio, se identificaron las estrategias competitivas que utilizan las microempresas del cantón Jipijapa y la incidencia en el desarrollo de sus actividades; se determinaron los factores de competitividad que influyen en el desarrollo micro empresarial, así como establecer estrategias competitivas que aporten al desarrollo micro empresarial en el cantón de Jipijapa. Para lograr La meta planteada se emplearon los métodos de investigación tales como: deductivo, inductivo, histórico, bibliográfico y estadístico, los mismos que aportaron en la búsqueda de información de las variables investigadas. Se utilizaron las técnicas de observación y encuesta, tomando como muestra a 326 microempresas de un universo de 2142 que existen en el cantón Jipijapa. Teniendo como resultado que las estrategias competitivas permiten en gran parte mejorar el nivel de ventas de los microempresarios de la zona urbana del Cantón Jipijapa, mantenerse vigentes a lo largo de los años en sus negocios, acaparar el mercado local sin tener que recurrir a otras ciudades aledañas, sean estas provinciales o regionales. Concluida esta investigación recomendamos a las entidades respectivas brindar capacitaciones a los microempresarios para así conocer más sobre el buen uso de las estrategias competitivas, que se verán reflejados en el logro de los objetivos estratégicos planteados.

Palabras claves: Liderazgo; Mercado; Calidad; Recursos Tecnológicos; Innovación.

\begin{abstract}
In case the research has been raised, the competitiveness strategies and the applications of micro enterprises of the Jipijapa canton, this is due to the main problem. How do the strategies of competition contribute to the strengthening of the micro enterprises of the Jipijapa canton? the competitive strategies used by the micro enterprises of the Jipijapa canton and the incidence in the development of their activities were identified; the competitiveness factors that influence microenterprise development were determined, as well as the competitive strategies that contribute to microenterprise development in the canton of Jipijapa. To achieve our stated goal, we used research methods such as: deductive, inductive, historical, bibliographic and statistical, which were provided in the search for information on the variables investigated, the observation and survey
\end{abstract}


techniques were used; a sample of 326 microenterprises of 2142 was taken, which is the total population. Once they obtained the results, the competitive accounts allowed the microentrepreneurs of the urban area of the Jipijapa Canton, to remain in their businesses over the years, monopolizing the local market without having to resort to others. Markets, be they provincial or national. To conclude that our research is in favor of the respective entities providing capacities for microentrepreneurs to achieve more competitive strategies and practices in their businesses, which is reflected in the increase in the level of sales, contributing to the achievement of the goals set out in each Microenterprise.

Keys words: Leadership; Market; Quality; Technological Resources; Innovation.

\section{Resumo.}

Caso a pesquisa tenha sido levantada, as estratégias de competitividade e as aplicações das microempresas do cantão de Jipijapa, isso se deve ao problema principal. Como as estratégias de competição contribuem para o fortalecimento das microempresas do cantão de Jipijapa? as estratégias competitivas utilizadas pelas microempresas do cantão de Jipijapa e a incidência no desenvolvimento de suas atividades foram identificadas; os fatores de competitividade que influenciam o desenvolvimento das microempresas foram determinados, assim como as estratégias competitivas que contribuem para o desenvolvimento de microempresas no cantão de Jipijapa. Para atingir nosso objetivo declarado, foram utilizados métodos de pesquisa como: dedutiva, indutiva, histórica, bibliográfica e estatística, os quais foram fornecidos na busca de informações sobre as variáveis investigadas, foram utilizadas as técnicas de observação e levantamento; foi colhida uma amostra de 326 microempresas de 2142, que é a população total. Uma vez obtidos os resultados, as contas competitivas permitiram aos microempresários da área urbana do Cantão de Jipijapa, permanecer em seus negócios ao longo dos anos, monopolizando o mercado local sem ter que recorrer a outros. Mercados, sejam eles provinciais ou nacionais. Conclui-se que nossa pesquisa é a favor das respectivas entidades que fornecem capacidades para os microempreendedores para alcançar estratégias e práticas mais competitivas em seus negócios, o que se reflete no aumento do nível de vendas, contribuindo para o alcance das metas estabelecidas em cada um deles. Microempresa.

Palavras chaves: Liderança; Mercado; Qualidade; Recursos Tecnológicos; Inovação. 


\section{Introducción.}

En el mundo actual de los negocios, las empresas deben ser competitivas, solo así lograran establecer en los mercados actuales donde existe gran rivalidad competitiva entre las empresas. Es por ello que, al fundar una organización o empresa, se debe elegir la mejor estrategia empresarial a seguir ya que esta es de suma importancia para alcanzar el éxito. (...) No puede ninguna empresa estar compitiendo sin misión, sin objetivos, sin metas y sin las estrategias necesarias para poder lograr esos objetivos, de esta manera es exigente para los propietarios o directivos que dicha decisión sea minuciosamente analizada (Monge, 2010).

Las micro, pequeñas y medianas empresas son agentes económicos claves para el desarrollo económico de un país, ya que buena parte de la población y de la economía dependen de su actividad y desempeño. En los países de la Unión Europea y de América Latina y el Caribe las pymes representan aproximadamente el 99\% del total de empresas, generan una gran parte del empleo (67\%) y actúan en una amplia variedad de ámbitos de la producción y de los servicios. (NU. CEPA, 2013)

El conjunto de microempresas que puede tener un país es fuente de progreso, ya que éstas en su conjunto impulsan la economía al consumir materias primas elaboradas como insumos para sus productos, igualmente se reconoce su poder de demanda de bienes intermedios, como por ejemplo los repuestos mecánicos. Como estos entes deben funcionar bajo ciertas condiciones mínimas de organización y contar con una planta física, también son demandantes de bienes de consumo durables como muebles, equipos de oficina, etc. (Guerrero Marin, 2001)

Actualmente el concepto de competitividad ha adquirido una gran presencia en la literatura tanto internacional como a escala local al momento de analizar el progreso económico tanto de países 
como de empresas. Comparativos internacionales permiten indagar que naciones — por medio de sus empresas - han brindado mejoras a sus ciudadanos a través del incremento en el nivel de vida. Por tanto, se reconoce que el nivel de competitividad internacional de un país se relaciona estrechamente con empresas altamente productivas que aprovechan las ventajas competitivas que genera el país por medio de sus instituciones, políticas, infraestructura y cultura. (Ibarra, Gonzalez, \& Demuner, 2017)

Existen muchas limitantes para que las microempresas puedan concretar su visión de empresas sólidas y competitivas en el mercado nacional e internacional, dentro de esta están la ilegalidad o informalidad y la dificultad de acceder a créditos. Factores indispensables para lograr un crecimiento sostenible y sustentable.

La mayoría de microempresas en el cantón Jipijapa son de carácter familiar, que se ejecutan con iniciativas de un ahorro doméstico, dedicándose al comercio de bienes y servicios, dentro las principales calles de la ciudad; la problemática radica en las limitantes que tienen para lograr ser competitivos está su actividad formal, las restricciones al momento de acceder a un crédito, las altas tasas de interés de un crédito micro empresarial y los requisitos que son para muchos microempresarios inalcanzables. Factores necesarios para conseguir un crecimiento sostenible y sustentable que les permita aportar al desarrollo económico del cantón, mejorar su calidad de vida y por ende a la generación de fuentes de empleos.

Una estrategia competitiva permite garantizar una posición ventajosa al resto de empresas que compiten en el mercado, en este marco, el presente trabajo de investigación es de gran importancia, porque permitió analizar las estrategias de competitividad para el fortalecimiento de las microempresas del cantón Jipijapa, fundamentado en sus normativas legales y en la asimilación de conocimiento adquiridos por el investigador, basado en sus variables tanto independiente como 
dependiente, además, busca analizar las estrategias de competitividad y como su aplicación fortalece a las microempresas del cantón de Jipijapa. En la actualidad los microempresarios no tienen claridad sobre el término "competitividad" y la importancia de utilizar estrategias que fortalezcan la parte interna y externa, que le permitan competir en menor desventaja con las empresas del mismo sector, permitiéndoles ser diferente a las demás en ciertos componentes como lo son calidad del producto o servicio, diversificación, potencial humano, procesos administrativos adecuados entre otros.

\section{Metodología.}

La aplicación metodológica en el trabajo de investigación, fue dado mediante los procedimientos lógicos, que direccionan el trabajo para su análisis y determinación de la situación actual. Los principales métodos y técnicas empleados para lograr lo planteado en este estudio fueron los siguientes:

Método descriptivo: Permitió poner de manifiesto la realidad de las microempresas del cantón Jipijapa. De esta forma se obtuvo información que caracteriza la problemática y la no aplicación de estrategias que permitan el posicionamiento y la ventaja competitiva.

Método deductivo: Consistió en demostrar de forma interpretativa varias conclusiones de los hechos observados en los diferentes entornos (interno y externo) correspondientes a las variables investigadas en este proyecto.

Método inductivo: Se dio a partir de la observación de los actores locales, con relación al presente estudio, este método reconoció trabajos similares relacionados con estrategias competitivas. 
Laura P. Muñiz-Jaime; José A. Franco-Yoza; Zoila M. Delgado-Gutierrez; Adela del Jesús Lucio-Pillasagua; Carlos R. Tumbaco-Tumbaco

Método estadístico: Este método consistió en manejar datos cualitativos y cuantitativos, mediante las técnicas primordiales y subsiguientes aplicadas en el presente trabajo de investigación a través de tablas y gráficos que permitieron obtener resultados concretos de la problemática a investigar.

\section{Técnicas}

La observación: Se fundamentó en saber con precisión todo lo referente al tema de proyecto investigativo: "Estrategias competitivas para el fortalecimiento de las microempresas del cantón Jipijapa"

La encuesta: Se estableció un cuestionario de preguntas, la misma que permitió obtener información directa de la utilización de estrategias competitivas en las microempresas de la zona urbana del cantón Jipijapa, dirigida a los propietarios de estas iniciativas empresariales.

\section{Población}

La población que se consideró dentro de este proyecto de investigación fue encuestas aplicadas a los Gerentes de las Microempresas del área urbana del cantón Jipijapa, la cual está constituida por 2.142 microempresas de acuerdo al informe final emitido por el Departamento de Catástrofe y avalúos del Municipio de Jipijapa, en base al Proyecto Multidisciplinario: "Fortalecimiento de la gestión del sector comercial y económico de los cantones Jipijapa, Paján y Puerto López” ejecutado por estudiantes del área de vinculación de la UNESUM de la Facultad Ciencias Económicas.

\section{Muestra}

Se calculó una muestra al total de microempresarios registrados en el último censo comercial: 326 fueron las personas encuestadas. 


\section{Resultados.}

Las estrategias en el sector empresarial sea esta desde la micro o hasta la gran empresa se han convertido en ejes principales de su desarrollo, hoy en día no hay sector empresarial que dentro de su estructura no adopte tácticas competitivas para mejorar sus ingresos y de este modo fortalecer sus inversiones.

Se detectó que las estrategias competitivas propuestas a corto plazo tienen resultados inmediato, pero para largo plazo no surten el mismo efecto en las microempresas, estas tienen que ser debidamente planificadas:

\section{Cuadro $N^{\circ}$ 1: Planificación de estrategias}

\begin{tabular}{|l|l|l|}
\hline Alternativa & Frecuencia & Porcentaje \\
\hline Planifica a corto plazo & 210 & $64 \%$ \\
\hline Planifica a largo plazo & 108 & $33 \%$ \\
\hline No planifican & 8 & $3 \%$ \\
\hline Total & $\mathbf{3 2 6}$ & $\mathbf{1 0 0} \%$ \\
\hline
\end{tabular}

Fuente: Microempresarios del cantón Jipijapa.

Elaborado por: Carlos Renán Tumbaco Tumbaco

Del mismo modo los encuestados creen en los porcentajes más representativos que la estrategia de diferenciación $(30 \%)$ y la estrategia de promoción publicitaria $(34 \%)$ son las estrategias que más aplican en sus negocios. Se observó que no planifica un 3\%.

Sobre los factores de la competitividad son muy esenciales para el fortalecimiento de las microempresas, se pudo constatar que de una manera empírica los microempresarios encuestados la 
Laura P. Muñiz-Jaime; José A. Franco-Yoza; Zoila M. Delgado-Gutierrez; Adela del Jesús Lucio-Pillasagua; Carlos R. Tumbaco-Tumbaco

han estado analizando y consideran que estas son un pilar importante para adquirir sus productos, ya que son las exigencias de sus demandantes:

\section{Cuadro $N^{\circ} 2$ : Factores de competitividad}

\begin{tabular}{|l|l|l|}
\hline Alternativa & Frecuencia & Porcentaje \\
\hline Hábitos de los clientes & 107 & $33 \%$ \\
\hline Las modas o las tendencias & 98 & $30 \%$ \\
\hline Los avances tecnológicos & 63 & $19 \%$ \\
\hline Otros factores & 58 & $18 \%$ \\
\hline Total & $\mathbf{3 2 6}$ & $\mathbf{1 0 0 \%}$ \\
\hline
\end{tabular}

Fuente: Microempresarios del cantón Jipijapa.

Elaborado por: Carlos Renán Tumbaco Tumbaco

Los principales factores de competitividad considerado por los encuestados para fortalecer sus inversiones microempresariales son los hábitos de los clientes, características propias de cada uno de los demandantes, es lo que hace que estos se conviertan en clientes permanentes del negocio y vuelvan contantemente a demandar los bienes o servicios que se brindan en cada uno de los locales, del mismo modo las modas y las tendencias así como los avances de la tecnología se convierten en los factores que dan la permanencia de las microempresas en el mercado local de Jipijapa.

La ventaja competitiva en las inversiones es fundamental para fortalecer los negocios, ya que esta hace ubicar a las empresas de cualquier índole e inversión en una posición estratégica para defenderse de las fuerzas competitivas ya que estas son un riesgo, sobre todo para mantener solida la base de sus clientes o demandantes: 
Estrategias de competitividad para el fortalecimiento de las microempresas del cantón Jipijapa

Cuadro $N^{\circ} 3:$ Ventaja competitiva a considerar un riesgo

\begin{tabular}{|l|l|l|}
\hline Alternativa & Frecuencia & Porcentaje \\
\hline Competitividad local & 29 & $9 \%$ \\
\hline Competitividad de cantones cercanos & 173 & $53 \%$ \\
\hline Competitividad provincial & 124 & $38 \%$ \\
\hline Competitividad nacional & 0 & $0 \%$ \\
\hline Total & $\mathbf{3 2 6}$ & $\mathbf{1 0 0} \%$ \\
\hline
\end{tabular}

Fuente: Microempresarios del cantón Jipijapa.

Elaborado por: Carlos Renán Tumbaco Tumbaco

Un porcentaje significativo (53\%) de los encuestados expresó que la ventaja competitiva de las microempresas que ofertan los productos o servicios de los cantones cercanos, específicamente Manta y Portoviejo son los que consideran un riesgo para la pérdida de sus clientes. Otros de los principales riesgos es la competitividad provincial (38\%), esencialmente la de la provincia del Guayas, en Guayaquil, donde muchas personas se movilizan a realizar compras por su cercanía a la ciudad de Jipijapa. Es evidente que la ventaja competitiva local (9\%) no les preocupa mayormente.

\section{Conclusiones.}

De acuerdo al trabajo investigativo se llegó a las siguientes conclusiones:

De la muestra aplicada a 326 microempresarios de la zona urbana del cantón Jipijapa, se pudo evidenciar que el 97\% si aplica estrategias competitivas, dentro de la que se destaca el enfoque de atraer cliente que es la base del éxito y el posicionamiento dentro del mercado local, mientras el 3\% no emplea por diversos factores tales como desconocimiento, factor dinero, y por infraestructuras 
inadecuadas que no permiten desarrollar de forma eficiente sus actividades empresariales; estos asumen que soportan presiones competitivas, limitantes para fortalecer su negocio en el mercado, poca diferenciación en los productos o servicios que ofrecen, lo que incide en el bajo desarrollo de sus negocios.

Los factores competitivos que influyen dentro del negocio según la encuesta realizada a los microempresarios, en un 33\% manifestaron conocer los hábitos de los clientes, esto les ayuda a conocer las necesidades de cada uno de los consumidores, permitiendo diversificar los productos y/o servicios que conlleva al crecimiento y rentabilidad de sus negocios; con el 30\% tenemos que las modas o tendencias permiten conocer el marketing adecuado que se debe impulsar para llamar la atención del cliente, que permita atraer más clientes; un 19\% de los encuestados, reconoce que los avances tecnológicos tienen el objetivo de crear plataformas novedosas, futuristas e innovadoras para ofrecer sus productos, hace que su industria crezca y no quedarse en el olvido, el 16\% tiene como aliado el análisis de la competencia, con un $0 \%$ tenemos que la gestión administrativa cuenta con un adecuado liderazgo y trabajo en equipo, generando una nueva cultura organizacional y un $0 \%$ hace referencia a los cambios culturales; por lo tanto deducimos que mediante la implementación correcta de todos estos elementos importantes, estos contribuyen al desarrollo micro empresarial y a su vez aporta a la sostenibilidad y sustentabilidad de sus negocios.

Entre las estrategias competitivas que los microempresarios emplean para que su micro empresa alcance el pleno desarrolló; tenemos con el 1\% la innovación, que es la presentación virtual de su negocio, es decir como estas microempresas generan mayores posibilidades de éxito, el 3\% prefiere hacer publicidad y promoción de su negocio, con la finalidad de captar la atención de potenciales nuevos clientes, así como sus clientes frecuentes; el 27\% lleva un control de su mercadería, permitiendo contar con el stock necesario de mercadería en sus negocios, así mismo mejora el 
servicio a sus clientes, ofreciendo productos diversos a los de sus competidores, dando alternativas de consumo en base a sus necesidades y recursos económicos; $\mathrm{y}$, un $28 \%$ se concentra en un grupo específico de clientes, alcanzando cubrir en gran parte este tipo de demandas que aportan al desarrollo micro empresarial del cantón Jipijapa.

Denotando entonces entre el principal obstáculo en el desarrollo de las microempresas es debido a la mala infraestructura que poseen, con espacios reducidos y que brindan poca estética a su identidad corporativa; además la escasa promoción de sus productos hace que decaiga el nivel de ventas, por eso se concluye que se deben aplicar las estrategias competitivas que permitirán en gran parte que los microempresarios de la zona urbana del Cantón Jipijapa fortalezcan sus actividades empresariales; y así, puedan mantenerse vigentes a lo largo de los años en sus negocios, acaparando el mercado local sin tener que recurrir a otros mercados, sean estos provinciales o nacionales, aun así es necesario recordarles que una buena sostenibilidad de un negocio también depende de su expansión, tanto personal, como su actividad micro empresarial.

\section{Recomendaciones.}

Se recomienda que las entidades como GAD Municipal, Cámara de Comercio entre otros, brinden capacitaciones a los microempresarios para así conocer más sobre las estrategias competitivas y aplicarlas en sus respectivos negocios, que se verán reflejados en el aumento del nivel de ventas, satisfacción no solamente del cliente sino del personal que labore dentro de estas; porque se sentirá motivado a trabajar de forma eficaz y eficiente día a día, aportando al logro de las metas planteadas en cada Microempresa.

Fortalecimiento a través de la aplicación correcta de estrategias competitivas, que permitan dinamizar las actividades micro empresariales, en base al apoyo por parte de un especialista 
(Ingeniero en Marketing), que direccione el norte al cual desean avanzar las microempresas con el uso óptimo de tácticas organizacionales eficientes; desarrollando la cultura de emprendimiento eficaz y oportuno en base a nuevas estrategias que permitan el crecimiento tanto empresarial y económico.

Establecimiento de encuestas periódicas (por lo menos trimestralmente), en la web para conocer las necesidades del consumidor, que ha demás permitirá la demostración de la diversificación de sus productos y/o servicios, promocionar descuentos y beneficios; que permitan a cada uno de los microempresarios que conforma la zona urbana del cantón Jipijapa, ser más competitivo en el mercado local, regional y porque no proyectarse a nivel nacional.

Que los agentes vendedores de las microempresas del cantón Jipijapa, mejoren la atención al cliente, para que estos clientes se lleven una grata acogida cuando visiten cualquier local comercial o de servicio, coadyuvando al buen trato que se merece, sea este local o que este de visita dentro de nuestra ciudad; y así esto permita la fidelidad de los clientes.

Que el GAD Municipal se preocupe por brindar un lugar estratégico a quienes ejercen el comercio minorista del cantón, lo que permitirá que exista comodidad tanto para el vendedor como para el consumidor, con la finalidad de que los pequeños microempresarios capten una gran afluencia de clientes, para que los recursos que se generan dentro de nuestra ciudad se queden dentro de la misma y no tengan que ir a comprar bienes o servicios a ciudades aledañas.

\section{Bibliografia.}

Guerrero Marin, M. A. (11 de julio de 2001). Gestiopolis. Obtenido de https://www.gestiopolis.com/papel-microempresas-desarrollo-economico/ 
Estrategias de competitividad para el fortalecimiento de las microempresas del cantón Jipijapa

Ibarra, M., Gonzalez, L., \& Demuner, M. (2017). Competitividad empresarial de las pequeñas y medianas empresas manufactureras de Baja California. Estudios Fronterizos, 18(35), 107130. Obtenido de Scielo.org.

Monge, E. C. (2010). Las estrategias competitivas y su importancia en la buena gestión de las empresas. Ciencias Económicas, 28(1), 247-276.

NU. CEPA. (2013). Cómo mejorar la competitividad de las PYMES en la Unión Europea y América Latina y el Caribe: propuestas de política del sector privado. Santiago, Chile: Cepal. 